\title{
Difficulties faced by second language learners of Arabic when translating Tamil sentences into Arabic
}

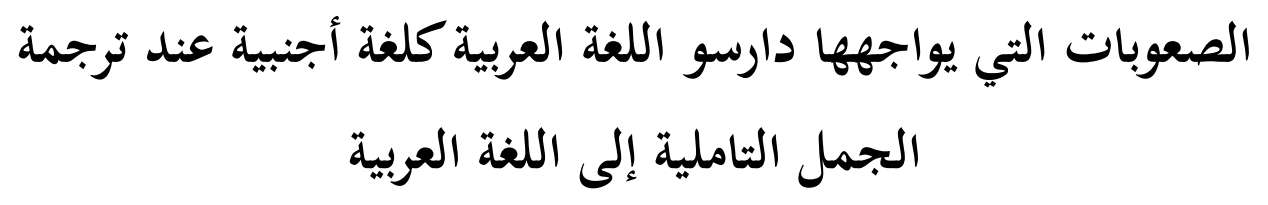

Muhammadu Sainulabdeen Zunoomy zunoomyzain94@gmail.com Department of Arabic Language, Faculty of Islamic Studies and Arabic Language, South Eastern University of Sri Lanka.

Mohammed Cassim Sithy Shathifa shathifa@seu.ac.lk Department of Arabic Language, Faculty of Islamic Studies and Arabic Language, South Eastern University of Sri Lanka.

• Received: 30.07.2020 • Accepted: 01.04.2021 • Published online: 05.05.2021

Abstract: The Muslims around the world use Arabic language for many functions. Likewise, Sri Lankan Muslims also use it in their daily worships and School \& University studies although they speak Tamil as a native language. According to this, the Learners of Arabic language as a foreign language face difficulties and make mistakes in identifying meanings of sentences and translating them into Arabic language. Therefore, this research aims to identify errors and difficulties faced by Arabic language learners when translating them. This research is based on: descriptive and analytical methodology. Primary data were gathered from interview and questionnaire. Interview was conducted with specialized students of second year from the department of Arabic language, Faculty of Islamic Studies and Arabic Language, South Eastern University of Sri Lanka and questionnaires were distributed to one hundred male and female general degree students of second year from the department of Arabic language. The sample of the study was chosen randomly among them. Secondary data were collected from books, research papers, articles, thesis and websites. MS Excel was used for data analysis. This research found that the Learners of Arabic language as a foreign 
Muhammadu Sainulabdeen Zunoomy, Mohammed Cassim Sithy Shathifa

language faced difficulties when translating in choosing appropriate words and determining their meaning. They also made mistakes in identifying subject, object, masculine and feminine, articles, adjective, genitive and in using appropriate conjunctions and prepositions.

Keywords: Translating Sentence, Sentence Pattern, Arabic Language, Tamil Language

الملخص: يستخدم المسلمون اللغة العربية لعدة وظائف في أنحاء العالم، هكذا مسلمو سريلانكا أيضا يستخدموفا في عباداقم اليومية ودراساقم المدرسية والجامعية رغم أن لغتهم الأم تكون اللغة التاملية. ولذا يواجه دارسو اللغة العربية لغة أجنبية في سريلانكا الصعوبات ويخطؤون في تحديد معاني الجمل وترجمتها إلى اللغة العربية. ولذا يهدف هذا البحث إلى تحديد الأخطاء والصعوبات التي يواجهها دارسو اللغة العربية لغة أجنبية عند ترجمتها. وللحصول على هذا الهدف، يعتمد هذا البحث على المنهج الوصفي التحليلي، واتبع الباحثان الطريقة الميدانية والمكتبية. وأما الطريقة الميدانية فهي تتمثل في المقابلات الشخصية والاستبانة. وبحري المقابلات مع الطلبة المتخصصين باللغة العربية في السنة الثانية وتوزع الاستبانة على مائة من الطلبة والطالبات من درجة البكالوريوس العامة من السنة الثانية من قسم اللغة العربية من كلية الدراسات الإسلامية واللغة العربية بجامعة جنوب شرق سريلانكا وتختار العينة عشوائيا منهم. وأما الطريقة المكتبية فهي كانت من الكتب والبحوث والمقالات والرسائل الجامعية والشبكات الإلكترونية. قد بستخدم البرنامج MS Excel لتحليل المعلومات. فتنتج هذا البحث أن دارسي اللغة العربية لغة أجنبية يواجهون الصعوبات عند الترجمة في اختيار الكلمات المناسبة وتحديد معناها وفي إضافة الكلمات لبناء الجمل الصحيحة وإضافة الجمل لتركيب الفقرات، كما أفم يخطؤون في تحديد الفاعل والمفعول والمذكر والمؤنث والتعريف والتنكير والنعت والمنعوت والمضاف والمضاف

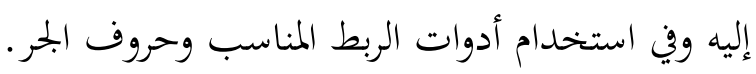
كلمات دلالية: ترجمة الجمل، نظام الجمل، اللغة العربية، اللغة التاملية 
Muhammadu Sainulabdeen Zunoomy, Mohammed Cassim Sithy Shathifa

المقدمة

تعريف عن اللغنين العربية والتاملية:

إن اللغة العربية هي لغة القرآن الكريم ولها فضل واسع وشرف مستمر من بداية نزول

القرآن البحيد على خاتم الأنبياء محمد صلى الله عليه وسلم. وهي عنوان الهوية، شرفها وبحدها وعمودها وعمادها وأساسها الراسخ المكين فهى تجسيد للهوية الإسلامية التي شرفها الله وأنزل

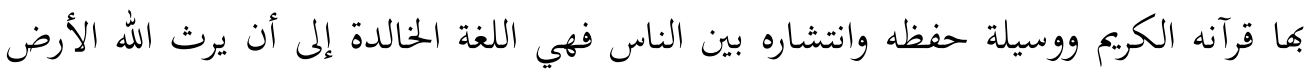
ومن عليها. تعتبر اللغة العربية من العائلة اللغوية السامية (Semitic) من أمهات اللغات المنتشرة حول العالم، وهى اللغة التي يتحدث بها أكثر من ملياري إنسان على سطح الأرض، كما أن اللغة العربية هي لغة الصيغ والإعراب والتصرف كما تختص بغنية أصواتا واشتقاق كلماتا

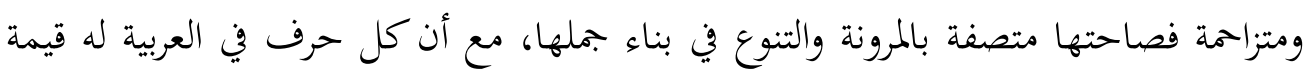

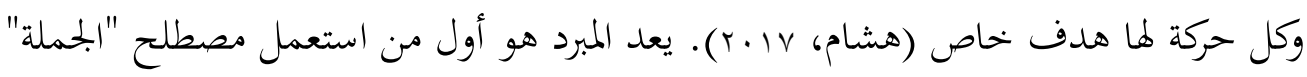

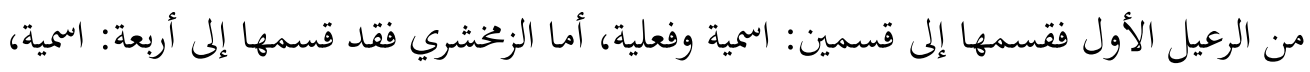
فعلية، شرطية و ظرفية، وهناك من قسمها إلى ثلاثة أقسام مثل ابن هشام ومن تبعه : اسمية ، فعلية و شرطية (مصلح، 17 (r). بناء على هذا، أن نظام الجملة في العربية تختص بترتيبها وتركيبها.

وأما اللغة التاميلية فهي أحد أطول اللغات الكلاسيكية في العالم أجمع. تم ذكرها في المرتبة العشرين في قائمة علم الأجناس باللغات الأكثر استخدامًا في جميع أنحاء العالم. تنتمي اللغة التاميلية إلى عائلة اللغات الدرافيدية ( السلطانة، و (ب). إن اللغة التاميلية تتضمن ثلاثة أقسام من نظام الجملة وهي الجملة البسيطة والجملة المركبة والجملة المعقدة. بناء على هذا، أن لكل لغة من هتين اللغتين نظاما خاصا بالجملة وتركيبها كما أن هناك عدة من أوجه التشابه والتناحر في نظام الجمل بين هتين اللغتين (صادفة وفرون، 10.ب). 
Muhammadu Sainulabdeen Zunoomy, Mohammed Cassim Sithy Shathifa

\section{الترجمة بين اللغات}

الترجمة كلمة عربية أصلية، التأرجمان والترجمان: المفسر للسان، الترجمان بالضم والفتح:

هو الذي يترجم الكلام، أي ينقله من لغة إلى لغة أخرى (ابن منظور، 1990). كما عرفها نيو

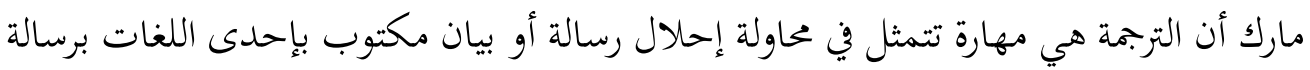

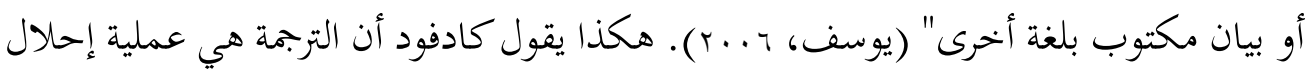
النص المكتوب بإحدى اللغات (يسميها اللغة المصدر) إلى نص يعادله مكتوب بلغة أخرى

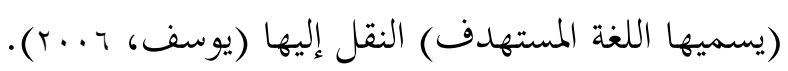

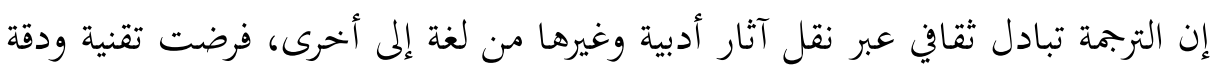

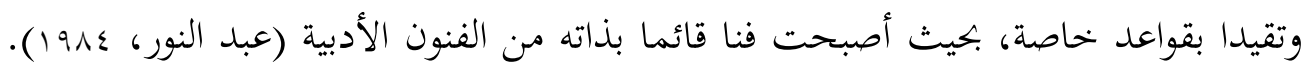
بناء على هذا، هي نقل المحتوى من لغة المصدر إلى لغة الهدف، والترجمة تعتبر فنا مستقلا بحد ذاته حيث أنه يعتمد على الإبداع والحس اللغوي والقدرة على تقريب الثقافات.

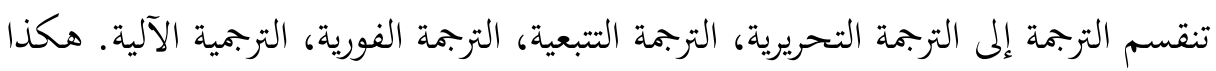
يقسم البعض الترجمة باعتبار النوعية إلى: النوع الأول - الترجمة ضمن اللغة الواحدة: وتعني هذه الترجمة أساسا لإعادة صياغة مفردات رسالة ما في إطار نفس اللغة. ووفقا لهذه العملية، يمكن ترجمة الإشارات اللفظية بواسطة إشارات أخرى في نفس اللغة، وهي تعتبر عملية أساسية نحو وضع نظرية وافية للمعنى، مثل عمليات تفسير القرآن الكريم. النوع الثاني - الترجمة من لغة إلى أخرى : وتعني هذه الترجمة ترجمة الإشارات اللفظية

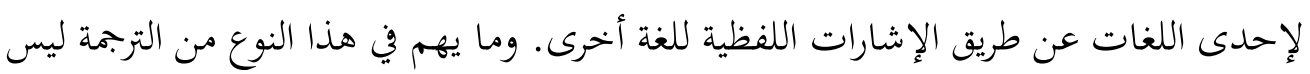

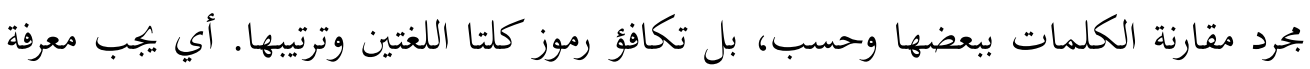
معنى التعبير بأكمله. النوع الثالث - الترجمة من علامة إلى أخرى : وتعني هذه الترجمة نقل رسالة من نوع معين من النظم الرمزية إلى نوع آخر دون أن تصاحبها إشارات لفظية، وبحيث يفهمها الجميع. فني 
Muhammadu Sainulabdeen Zunoomy, Mohammed Cassim Sithy Shathifa

البحرية الأمريكية على سبيل المثال، يمكن تحويل رسالة لفظية إلى رسالة يتم إبلاغها بالأعلام،

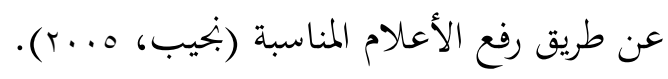

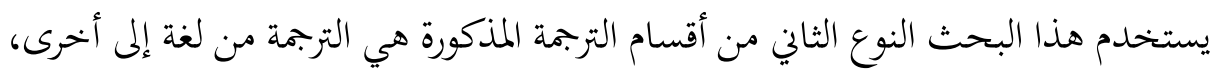
لأن هذا البحث يحاول أن يحدد الأخطاء والصعوبات التي يواجهها دارسو اللغة العربية لغة أجنبية عند ترجمة الجمل. كما أن اللغتين العربية والتاملية تنتميان إلى العائلة اللغوية المختلفة، فعملية الترجمة ليس بأمر سهل. لأفما تتضمنان القواعد المتنوعة في بناء الجمل وتركيب العبارات. حين أشار سليمان بن عمر السحيباني عن الدراسات التقابلية بين نظام الجمل للغات فقال إن

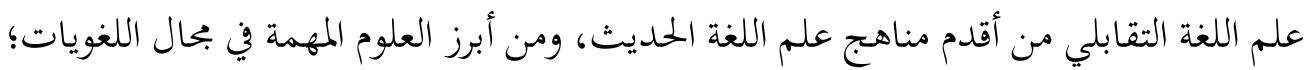
إذ يهتم بدارسة النظام اللغوي في مختلف اللغات، ومقابلتها بعضها ببعض، وإبراز مواطن التشابه والاختلاف بين اللغات (السحيباني، 10.r). حسب هذه القضية، إن هذا البحث حلّد الصعوبات والأخطاء في ترجمة الجمل التاملية إلى العربية خلال الدراسة التقابلية بين اللغات.

\section{الجملة في اللغنين العربية والتاملية}

أما الجملة فهي الصورة اللفظية الصغرى للكالام المفيد في أية لغة من اللغات، وهي المركب الذي يبين المتكلم به صورة ذهنية تألفت أجزاؤها في ذهنه، ثم هي الوسيلة التي تنقل ماجال في ذهن المتكلم إلى ذهن السامع. والجملة التامة التي تعبر عن أبسط الصور الذهنية التامة التي يصح السكوت عليها، تتألف من ثلاثة عناصر رئيسية، هي: المسند إليه، والمسند، والاستناد. إن في كل لغة جملا، ولكل منها نظاما خاصا. بناء على هذا ، إن الجملة العربية لما صورتان : 1 - اسمية ب - فعلية، فالجملة الاسمية

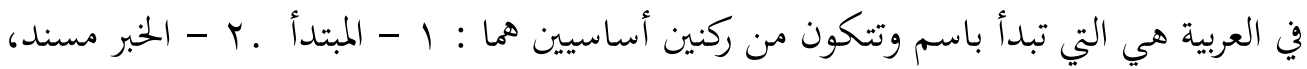
فعند ذكر المبتدأ ( المسند إليه ) لا بد من تحديد الخبر ( المسند ) ليتم المعنى ، وحكم كل منهما

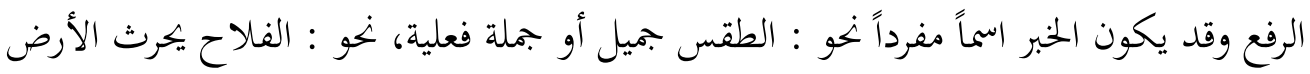

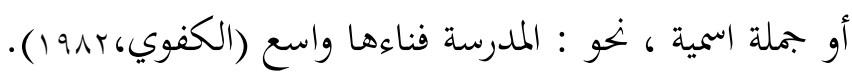


وأوسع من ذلك في وسائل التفرقة أن اللغة العربية تسمح بابتداء الجملة بحرف الجر وتؤدي

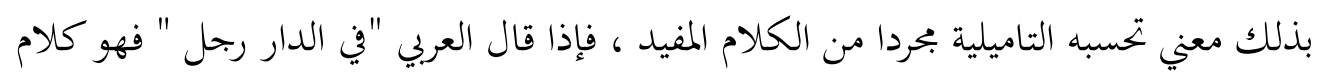
مفيد ، وتقديم الجار والبحرور فيه مقصود ، لأنه يشتمل على تنبيه لا يؤديه هذا الأداء في قول

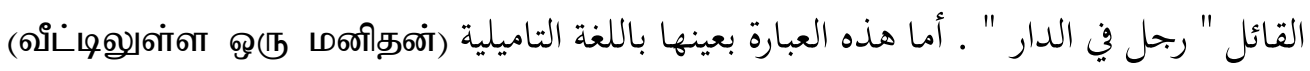
/oru maintain ve:ddil/ في بداية الجملة بخلاف اللغة العربية التي لا يجوز الابتداء بنكرة ، إلا بمسوغ ، مثال: ( | / oru maintain ve:ddil irukkinra:n/ (மனிதன் வீட்டில் இருக்கின்றான் رجل ".

أما الصورة الثانية للجملة في العربية فهي الجملة الفعلية التي تبدأ بفعل وتعتمد ترتيبا أساسيا من فعل ( مسند ) ، وفاعل ( مسند إليه ) ومفعول به إذا كان الفعل متعدياً

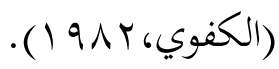

أما اللغة التاميلية فتختص نوعا واحدا من الجمل هي الاسمية ، وهي تنقسم إلى ثلاثة (கூட்டு வாக்கியம்) ، أقسام : البحملة البسيطة / tani va:kkiyam/ (தனி வாக்கியம்) /kalappu va:kkiyam/ (கலப்பு வாக்கியம்) ، الجملة المركبة" / ko:ttu va:kkiyam/

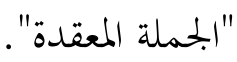

فالجملة البسيطة في اللغة التاميلية تتكون من جزء واحد ذو معنى محدد ، وتوصف كجزء

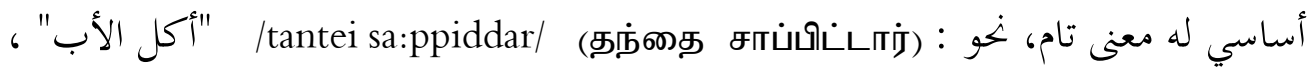
"أكل الأب /tantai iravunawai sa:ppiddar/ (தந்தை இரவுணவை சாப்பிட்டார்) العَشَاء". وهذا لا يعني أن الجملة البسيطة قصيرة ـ فهناك جمل طويلة توصف بأها جمل بسيطة ، சிறுவன் பாடசாலையிலிருந்து தந்தையுடன் பேரூந்தில் வீட்டுக்கு ) : /siruwan pa:dasa:lailiruntu tantaiudan pe:runtil we:ttuku wanta:n/ (வந்தான் "جاء الولد من المدرسة إلى البيت مع أبيه بالحافلة" (Karunakaran,2000). 
Muhammadu Sainulabdeen Zunoomy, Mohammed Cassim Sithy Shathifa

أما الجملة المركبة فتتكون في اللغة التاميلية من جملتين بسيطتين أو أكثر ترتبط بعضها um, o:, alltu, a:na:1,/ (உம், ஓ, அல்லது, ஆனால், ஆகவே) : ببعض بروابط، مثل

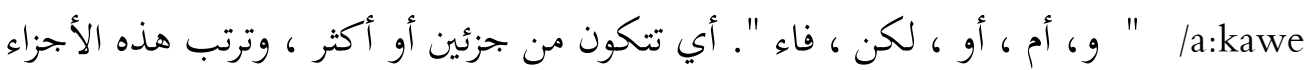

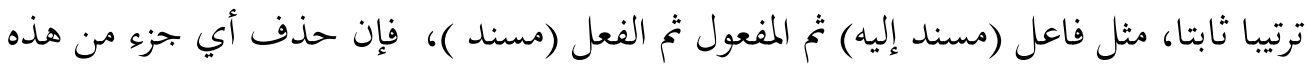
நாம் தேட்டத்திற்கு செல்வோம் அல்லது ) الجملة، يؤدي إلى تغيير المعنى، مثلا: na:m toddattikku selwo:m allatu kadatkaraiku / (கடற்கரைக்குச் செல்வோம்

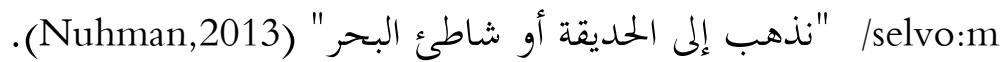
|kalappu va:kkiyam/ (கலப்பு வாக்கியம்) أما من الجمل في اللغة التاميلية فهى "الجملة المعقدة" التي تحتوي أكثر من فعل، وتتكون من جملتين : أساسية، وثانوية، تتضمن فكرة

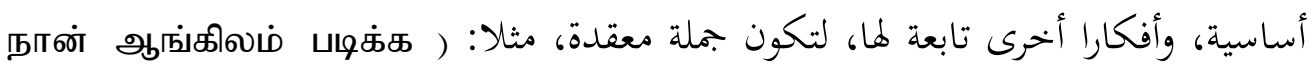
"أردت أن أدرس اللغة /na:n a:ngilam padikka virumpinen/ (விரும்பினேன் الإنحليزية" (Karunakaran,2000).

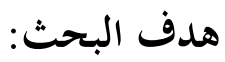

يهدف هذا البحث إلى تحديد تحديات ترجمتها عند دارسي اللغة العربية لغة أجنبية. بناء على هذا، إن دارسي اللغة العربية لغة أجنبية يواجهون الصعوبات وبخطؤون في تحديد معاني الجمل وترجمتها إلى اللغة العربية. ولذا يحدّد هذا البحث الأخطاء والصعوبات التي يواجهها لتهيه دارسو اللغة العربية لغة أجنبية عند ترجمة الجمل التاملية إلى اللغة العربية. حدود البحث:

الحد الموضوعي: ترجمة خمس جمل تاملية من الجمل البسيطة والجمل المركبة والجمل المعقدة

$$
\text { حد المى اللغة العربية. }
$$


Muhammadu Sainulabdeen Zunoomy, Mohammed Cassim Sithy Shathifa

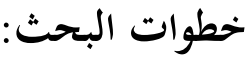

إن هذا البحث يحاول أن يحدد الأخطاء والصعوبات عند ترجمة الجمل التاملية إلى اللغة العربية لدى دارسي اللغة اللعربية لغة أجنبية. لذا، هذا البحث يتحدث عن اللغتين المختارتين لمعرفة حالتهما اللغوية، ثم يعالج ترجمة اللغات مع أنواعها المتنوعة لإكمال الإطار النظري له، تاليا يتكلم تركيب الجمل في اللغتين العربية والتاملية لإتاحة معرفة استرشادية للقارئ، بعد ذلك يعالج

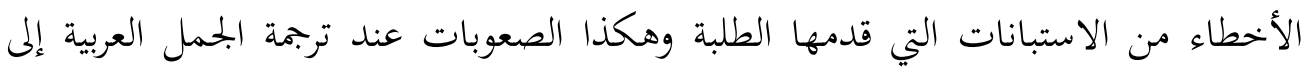
العربية، أخيرا يقدم التوصيات المناسبة لحلول الصعوبات.

\section{منهجية البحث}

إن هذا البحث يتناول ترجمة الجمل بين اللغتين بمختلف العائلة اللغوية، ولذا يستخدم هذا البحث وسيلة تحليل بالمنهج الوصفي التحليلي للحصول على المعلومات اللازمة كما أنه يعالج بالدراسة التقابلية. وتحصل المعلومات من الطريقة المكتبية والميدانية. وأما الطريقة المكتبية فهي تكون من الكتب والبحوث والمقالات والرسائل الجامعية و الشبكات الإلكترونية. أما الطريقة الميدانية فهى تتمثل في المقابلات الشخصية والاستبانة. وبتحري المقابلات مع الطلبة المتخصصين باللغة العربية في السنة الثانية وتوزع الاستبانة على مائة من الطلبة والطالبات من السنة الثانية من قسم اللغة العربية وتختار العينة عشوائيا من السنة الثانية. ومن أجل الحصول على نتائج علمية دقيقة، تستخدم الطريقة النوعية والكمية من المنهج الوصفي الإحصائي في استنتاج الاستبانات بمساعدة برنامج التكنولوجي الحديث MS Excel.

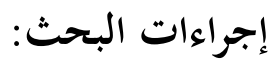

تقع الإجراءات المنهجية للدراسة وتحديد بجتمعها وعينتها، وأدواتما من حيث بنائها وتقنينها

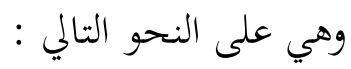

منهج الدراسة : استخدم الباحث في الدراسة الحالية المنهج الوصفي التحليلي لمعرفة درجة الصعوبات والأخطاء التي يواجهها دارسو اللغة العربية لغة أجنبية في تحديد معاني الجمل وترجمتها بين اللغتين العربية والتاميلية وذلك من خلال الاستجابات التي تم تفريغها من أداة الدراسة. 
Muhammadu Sainulabdeen Zunoomy, Mohammed Cassim Sithy Shathifa

مجتمع الدراسة : وقد نوعت عينة الدراسة إلى نوعين رئيسيين :أولا المشاركون في الاستبانة : وقد شارك . . 1 من طالب وطالبة من السنة الثانية من قسم اللغة العربية في كلية الدراسات الإسلامية واللغة العربية بجامعة جنوب شرق سريلانكا. ثانيا المشاركون في المقابلات الشخصية: وقد شارك عشرة طلبة من المتخصصين باللغة العربية في السنة الثانية في كلية الدراسات الإسلامية واللغة العربية بجامعة جنوب شرق سريلانكا.

طرق جمع البيانات وتحليلها: وزع الباحثان الاستبانات على جميع بحتمع الدراسة، وقد استبعد

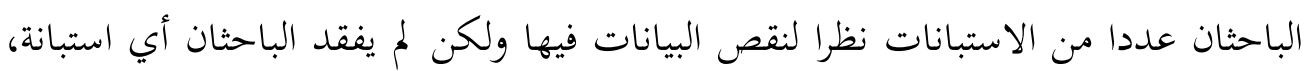

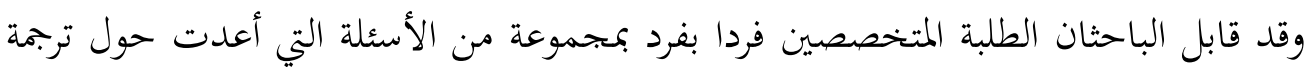
الجمل التاميلية إلى اللغة العربية. والجحول (1) يوضح العدد الموزع ، والعدد المفقود، والعدد المستبعد، والعدد النهائي الذي تمت عليه عملية التحليل .

الجدول | : أعداد الاستبانات الموزعة والمفقودة والمستبعدة والمتبقى منها

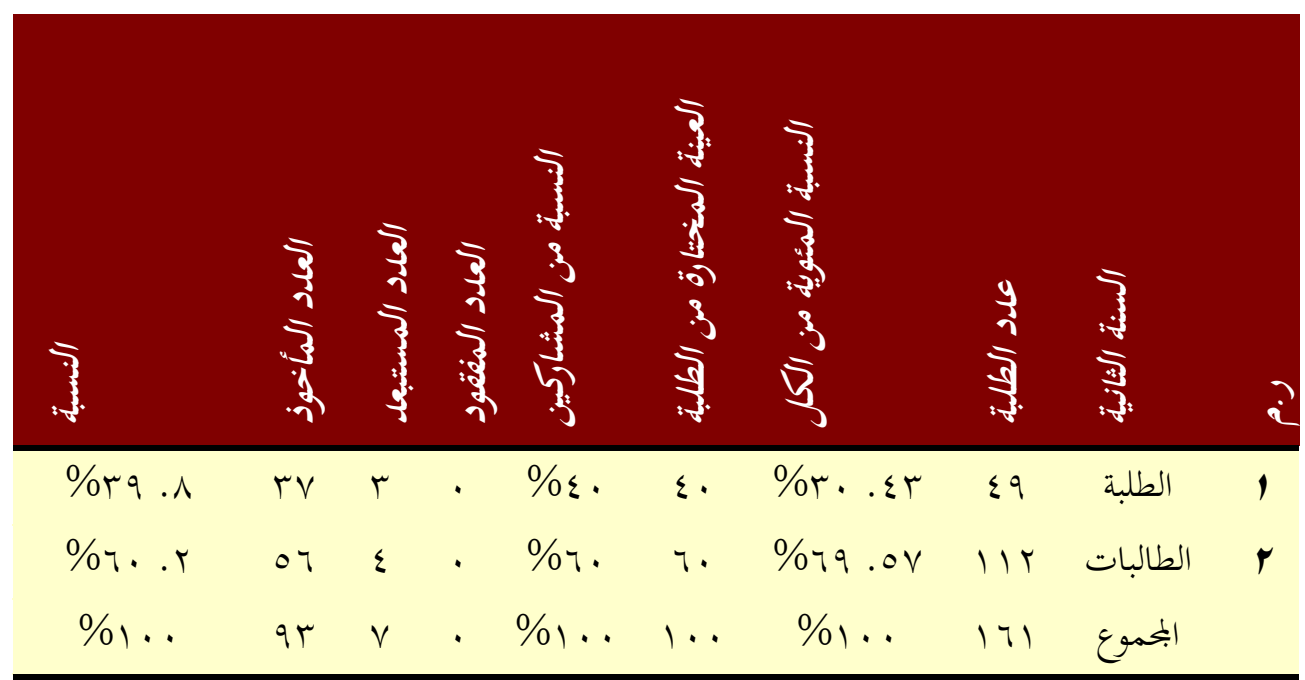

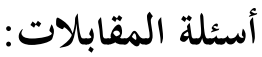

ـ ـ ـ أسباب تعلم اللغة العربية؟ 
Muhammadu Sainulabdeen Zunoomy, Mohammed Cassim Sithy Shathifa

$$
\begin{aligned}
& \text { ץ. هل تريد أن تتعلم اللغة العربية؟ } \\
& \text { ץ. ما رأيك في مواصلة دراسة البكاليوريوس؟ } \\
& \text { ع. ـ هل أنت متعلق بالترجمة؟ } \\
& \text { ه. ما هى الجهود التي قدمتَ في بحال الترجمة؟ } \\
& \text { 7. تكلم عن نظام الجمل التاميلية بوضوح. } \\
& \text { V. تحدث عن نظام الجمل العربية بوضوح. } \\
& \text { ^. ما هى الصعوبات التي تواجهها عند ترجمة الجمل بين العربية والتاميلية؟ }
\end{aligned}
$$

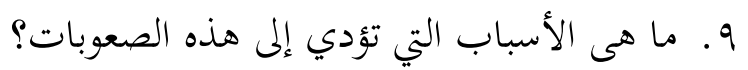

$$
\begin{aligned}
& \text { • } 1 \text { ـ ما هى التوصيات لحلول هذه الصعوبات؟ }
\end{aligned}
$$

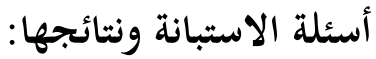

النوع الأول : tani va:kkiyam/ தனி வாக்கியங்கள்/ (الجمل البسيطة)

1. முஹம்மத் ஒரு ஆசிரியர் ஆவார்

\{محمد مدرس (Muhammat oru a:siriyar a:va:r)

2. நான் என்னுடைய வீட்டுக்குச் சென்றேன்.

(Na:n ennudaya ve:dduku senren)

3. தந்தை எப்போது சந்தைக்குப் போகிறார்?

(متى يذهب الأب إلى السوق؟ (Tantai eppodhu santaikup po:hira:r?)

4. அஹ்மத் உன்னோடு பேசமாட்டான்.

\{ لا يتكلم أحمد معك و (Ahmed unnodu pe:sama:dda:n)

5. இது ஒரு மிக அழகான பேனை இல்லை.

\{هذا ليس بقلم جميل جدأ (Idhu oru azhahana penai illai) 
Muhammadu Sainulabdeen Zunoomy, Mohammed Cassim Sithy Shathifa

\section{نتائج الدراسة وتحليلها}

أخطاء ترجمة الجمل التاملية إلى اللغة العربية:

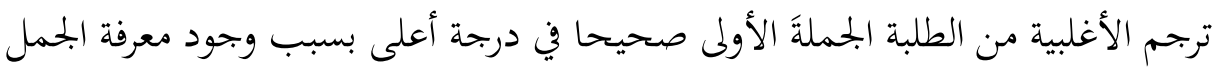

البسيطة، وأما الأقل منهم (ثلاثة) فأخطأوا في قواعد النحو العربي. وترجم الأغلبية من الطلبة

الجملة الثانية صحيحا في درجة متوسطة بسبب وجود معرفة الجمل البسيطة، وأما الأقل منهم (عشرون) فأخطأوا في قواعد النحو وستة عشر في تحديد الأزمنة. وترجم الأغلبية من الطلبة الجملة الثالثة صحيحا في درجة متوسطة بسبب وجود معرفة الجمل البسيطة، وأما الأقل منهم تسعة وثلاثون فأخطأوا في قواعد النحو العربي وثلاثة في عناية نظام الجمل العربية وثمانية في تحديد الأزمنة.

وترجم الأغلبية من الطلبة الجملة الرابعة صحيحا في درجة متوسطة بسبب وجود معرفة

الجمل البسيطة، وأما الأقل منهم سبعة وثلاثون فأخطأوا في قواعد النحو العربي وخمسة في عناية نظام الجمل العربية وأحد في تحديد الأزمنة. وترجم الأقلية من الطلبة الجمملة الخامسة صحيحا في درجة منخفضة، وأما الأكثر منهم خمسة وخمسون فأخطأوا في قواعد النحو العربي

وواحد وثلاثون في عناية نظام الجمل العربية، ويوضحها الشكل (1).

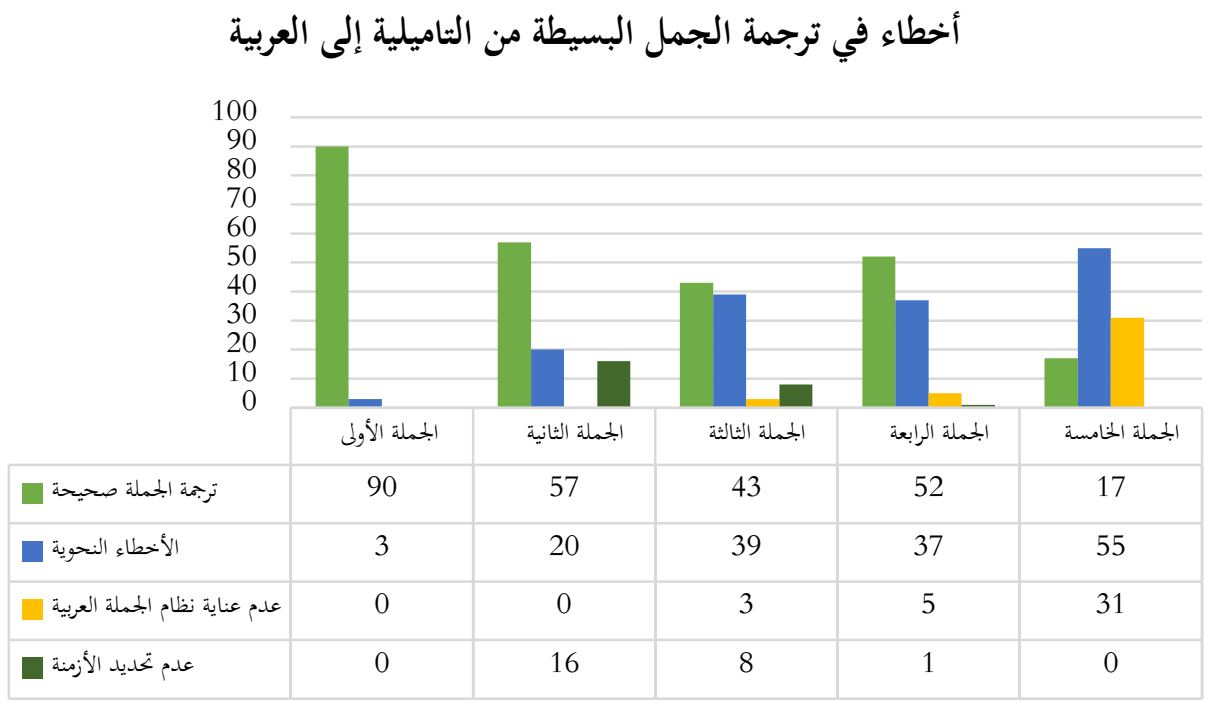

الشكل 1: أخطاء في ترجمة الجمل البسيطة من التاميلية إلى العربية (1-ه)

Indonesian Journal of Arabic Studies, Volume (3), Issue (1), May 2021 
النوع الثاني : النفا

6. நானும் அவனும் வீட்டுக்குப் போனோம்.

\{ أنا وهو ذهبنا إلى البيت (Na:num avanum ve:ddukkup po:no:m)

7. நீ பார்த்த அல்லது கேட்ட விஷயத்தைப் பற்றிச் சொல்.

\{ (Ne: pa:rtta alladhu kedda visayattai patti so:1)

8. இந்த நூலை பலமுறை வாசித்தேன்; ஆனால் எனக்கு ஒன்றும் புரியவில்லை.

\{قرأت هذا الكتاب عدة مرات ولكن لم أفهم شيئ (Inta no:lai palamurai paditten; a:na:1 enaku onrum puriyavillai)

9. ஆசிரியர் வந்திருக்கின்றார்; ஆகவே, இன்று அவர் கற்பிப்பார். \{يجضر المدرس فيعلّم اليوم) (a:siriyar vantirukkira:r; a:kave inru avar katpippa:r)

10. நான் தாங்கி எழும்பி சாப்பிட்டு விட்டு அலுவலகத்திற்கு சென்றேன். \{ (Na:n to:ngi ezhumpi sa:ppiddu viddu aluvalahattitku senren)

أخطاء ترجمة الجمل التاملية إلى اللغة العربية:

ترجم اثنان وعشرون طالبا الجملة السادسة صحيحا، وفي حين أخطأ الأغلبية من الطلبة

بسبب عدم عناية نظام الجمل العربية في درجة عالية، وأما الأقل منهم ثلاثة وعشرون فأخطأوا في قواعد النحو العربي وخمسة عشر في تحديد الأزمنة واثنان بسبب عدم وجود المعرفة في إضافة الجمل العربية.

وترجم ستة عشر طالبا الجملة السابعة صحيحا، وفي حين أخطأ الأغلبية من الطلبة

بسبب عدم وجود المعرفة في إضافة الجمل العربية في درجة عالية، وأما الأقل منهم تسعة عشر فأخطأوا بسبب عدم عناية نظام الجمل العربية وأربعة عشر في قواعد النحو العربي واثنان في تحديد الأزمنة. 
Muhammadu Sainulabdeen Zunoomy, Mohammed Cassim Sithy Shathifa

وترجم أربعة وعشرون طالبا الجملة الثامنة صحيحا، وفي حين أخطأ الأغلبية من الطلبة

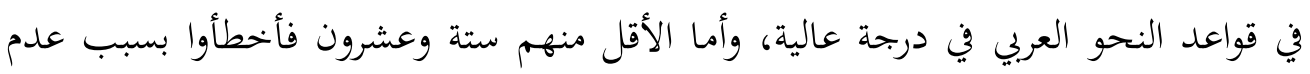
وجود المعرفة في إضافة الجمل العربية وتسعة عشر بسبب عدم عناية نظام الجمل العربية وثلاثة بعدم تحديد الأزمنة.

وترجم خمسة وعشرون طالبا الجملة التاسعة صحيحا، وفي حين أخطأ الأغلبية من الطلبة بسبب عدم وجود المعرفة في إضافة الجمل العربية في درجة عالية ، وأما الأقل منهم ثلاثة وعشرون فأخطأوا في تحديد الأزمنة واثنان وعشرون بعدم عناية نظام الجمل العربية وأربعة وعشرون في قواعد النحو العربي.

وترجم خمسة عشر طالبا الجملة العاشرة صحيحا، وفي حين أخطأ الأغلبية من الطلبة بسبب عدم وجود المعرفة في إضافة الجمل العربية في درجة عالية، وأما الأقل منهم ثلاثة وثلاثون فأخطأوا بعدم عناية نظام الجمل العربية وثمانية عشر في قواعد النحو العربي وثمانية في تحديد

الأزمنة، ويوضحها الشكل (r).

\section{أخطاء في ترجمة الجمل المركبة من التاميلية إلى العربية}

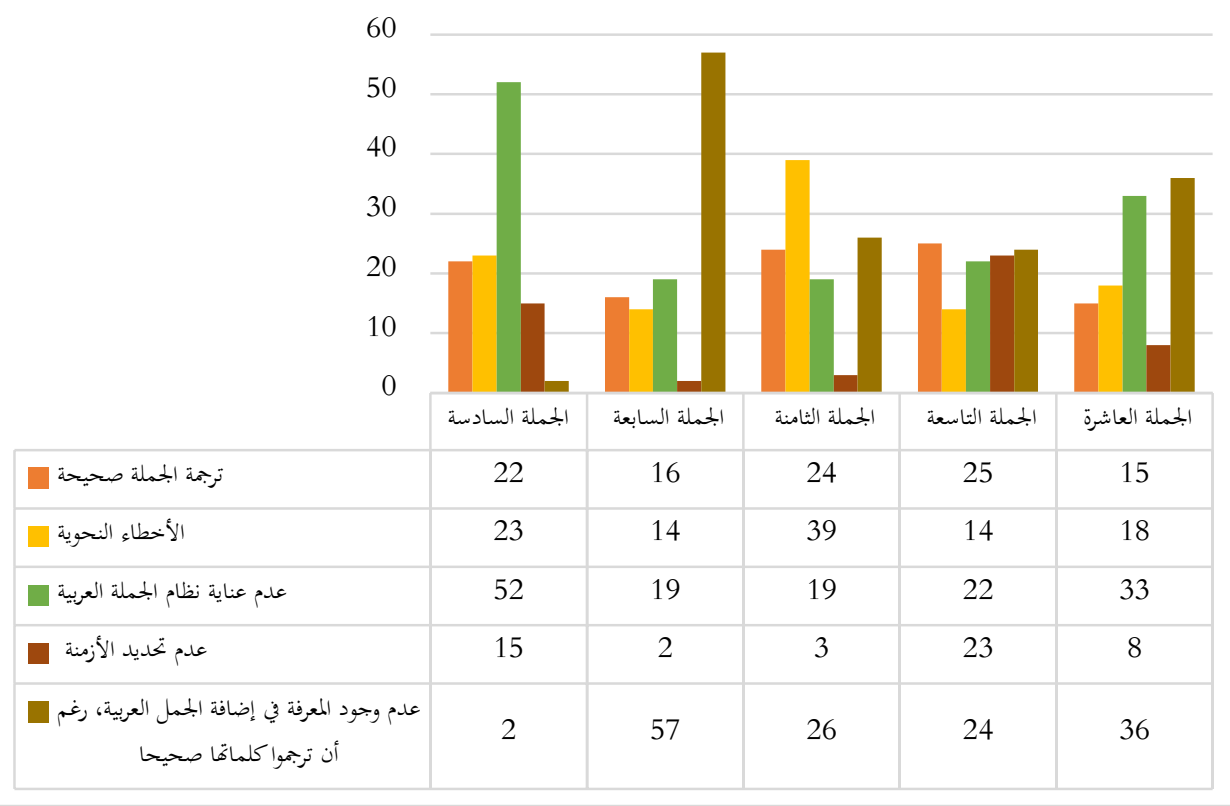

الشكل ץ: أخطاء في ترجمة الجمل البسيطة من التاميلية إلى العربية (ף- + )

Indonesian Journal of Arabic Studies, Volume (3), Issue (1), May 2021 
Muhammadu Sainulabdeen Zunoomy, Mohammed Cassim Sithy Shathifa

النوع الثالث :

11. இது நான் படிக்கின்ற பல்கலைக்கழகம்.

\{هذه جامعة أدرس فيها (Idhu na:n padikkira palhalaikkazhaham)

12. நான் பத்து வருடம் வாழ்ந்த இந்த ஊர் எனக்கு மிகவும் பிடித்தது. \{ (Na:n pattu varudam va:zhnta inta o:r enaku mihavum pidittadhu)

13. நான் யாரிடமிருந்து அன்பை எதிர்பார்த்தேனோ, அவர் எனக்கு அன்பு காட்டவில்லை.

\{ (Na:n ya:ridamiruntu anpai edhirpa:rteno:, avarenaku anpu ka:ddavillai)

14.அப்பா வந்திருக்கிறார் எனும் செய்தி எனக்கு இப்போது தான் தெரியும். \{ Appa vantirukkira:r enum seidhi enaku ippodhu tha:n terium)

15. ஹாமித் வெளிநாடு போனதைப் பற்றி நான் கேள்விப்படவில்லை. \{ما سمعت عن ذهاب حامد إلى الخارج (Ha:midh velina:du po:nadhai patti na:n kelvippdavillai)

أخطاء ترجمة الجمل التاملية إلى اللغة العربية:

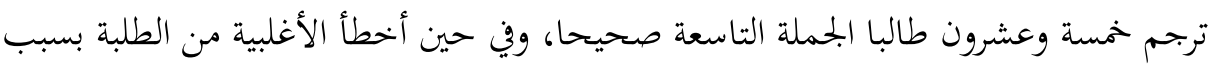

عدم وجود المعرفة في إضافة الجمل العربية في درجة عالية ، وأما الأقل منهم ثلاثة وعشرون فأخطأوا بسبب عدم تحديد الأزمنة واثنان وعشرون بعدم عناية نظام الجمل العربية وأربعة وعشرون بعدم معرفة قواعد النحو العربي.

وترجم ثمانية عشر طالبا الجملة الحادية عشرة صحيحا، ويف حين أخطأ الأغلبية من الطلبة

بسبب عدم عناية نظام الجمل العربية في درجة عالية، وأما الأقل منهم عشرون فأخطأوا في قواعد النحو العربي وتسعة عشر بسبب عدم وجود المعرفة في إضافة الجمل العربية وخمسة بعدم تحديد الأزمنة. وترجم تسعة عشر طالبا الجملة الثانية عشرة صحيحا، وفي حين أخطأ الأغلبية من الطلبة بسبب عدم عناية نظام الجمل العربية في درجة عالية، وأما الأقل منهم تسعة وعشرون فأخطأوا في 
Muhammadu Sainulabdeen Zunoomy, Mohammed Cassim Sithy Shathifa

قواعد النحو العربي وأربعة عشر بسبب عدم وجود المعرفة في إضافة الجمل العربية وتسعة بعدم تحديد

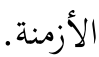

وترجم واحد وعشرون طالبا الجملة الثالثة عشرة صحيحا، وفي حين أخطأ الأغلبية من الطلبة بسبب عدم وجود المعرفة في إضافة الجمل العربية في درجة عالية، وأما الأقل منهم سبعة وعشرون

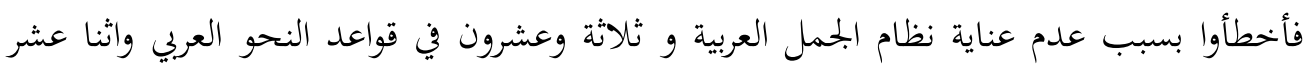
بعدم تحديد الأزمنة.

وترجم سبعة عشر طالبا الجملة الرابعة عشرة صحيحا، وفي حين أخطأ الأغلبية من الطلبة

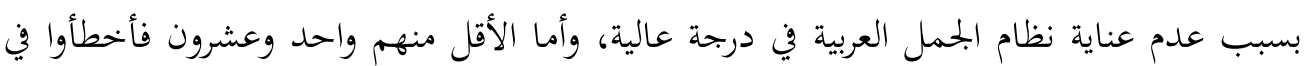

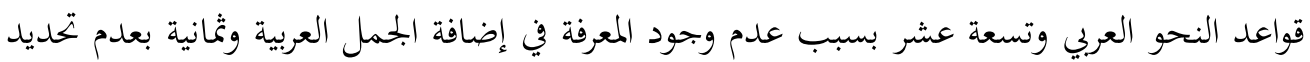
الأزمنة.

وترجم ثلاثة وعشرون طالبا الجملة الخامسة عشرة صحيحا، وفي حين أخطأ الأغلبية من الطلبة بسبب عدم وجود المعرفة في إضافة الجمل العربية في درجة عالية، وأما الأقل منهم ستة وعشرون

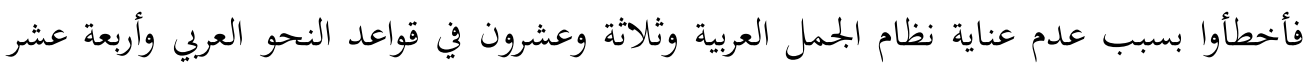
بعدم تحديد الأزمنة، ويوضحها الشكل (r).

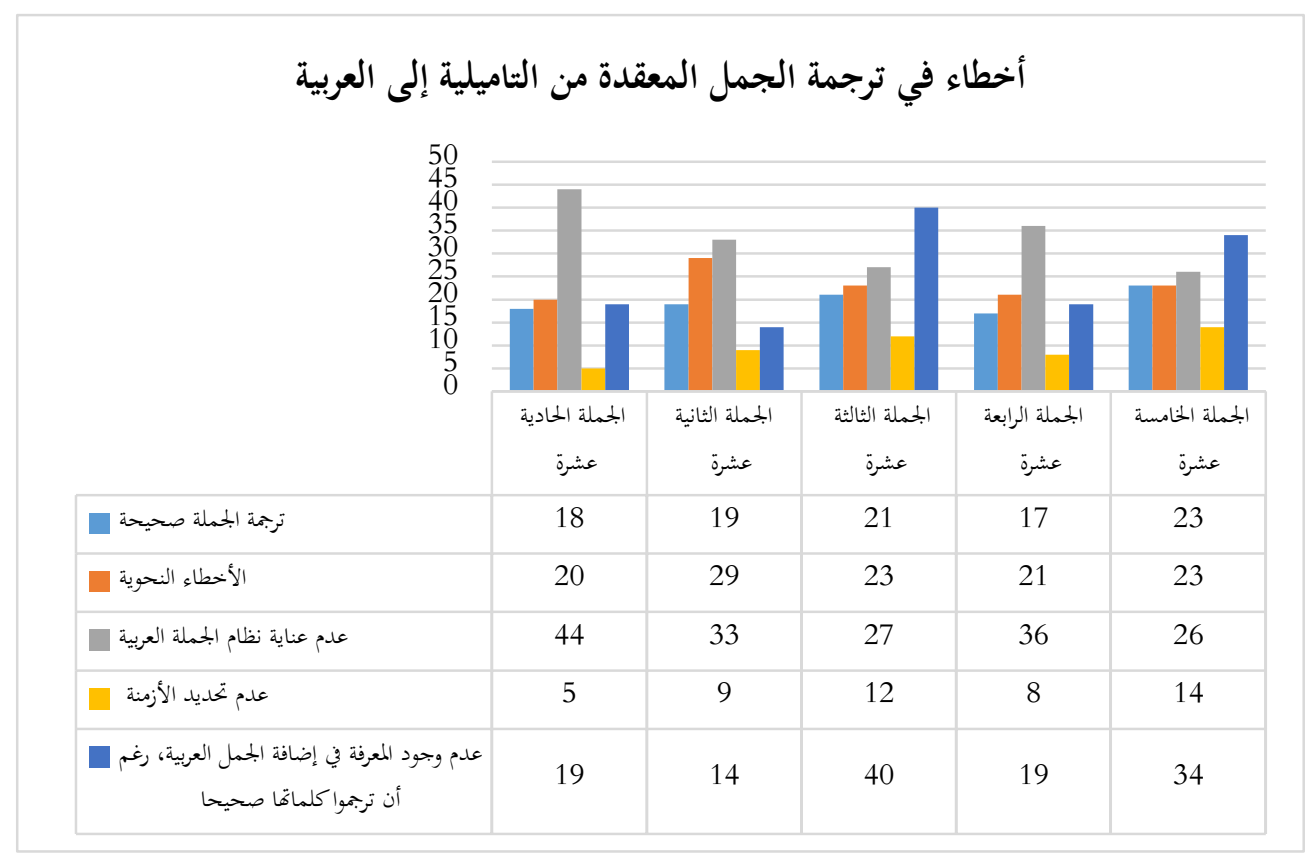

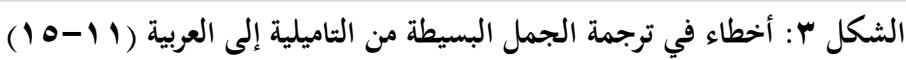

Indonesian Journal of Arabic Studies, Volume (3), Issue (1), May 2021 
Muhammadu Sainulabdeen Zunoomy, Mohammed Cassim Sithy Shathifa

إن هناك صعوبات كثيرة تتعلق بالترجمة من اللغة الأم إلى اللغة الأجنبية، منها ما هو

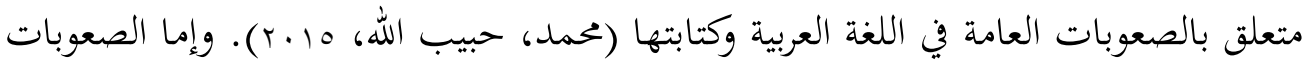
الرئيسية في الترجمة فهي الكفاءة اللغوية وإضافة الجمل (هشام ومناس وعليار، V لا.ب) والمعرفة عن سياق الجمل (عليار وصادفة وفرون، عا.ب). بناء على هذا، يبين الجحدول (r) الصعوبات التى واجهها الطلبة عند ترجمة هذه الجمل المذكورة إلى العربية. الجدول ب : صعوبات في ترجمة الجمل التاميلية إلى اللغة العربية

\begin{tabular}{|c|c|c|c|c|c|}
\hline رافضر & 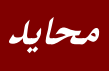 & 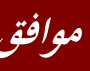 & موافت تماما & الصعوبات & ק. \\
\hline 9 & $r \varepsilon$ & r & IV & عدم التعلق بالترجمة & 1 \\
\hline rᄉ & $r \varepsilon$ & $\mu$ & 1. & عدم وجود المعرفة عن نظام الجمل في التاملية & r \\
\hline 9 & $r$. & r & rی & قلة ثروة الكلمات & $r$ \\
\hline 7 & rq & rq & 19 & عدم وجود المعرفة عن قواعد اللغة العربية & $\varepsilon$ \\
\hline iv & rV & rA & ri & الضعف في الكتابة بالعربية & 0 \\
\hline 7 & $r \varepsilon$ & $r$. & rt & الضعف في إضافة الجمل & 7 \\
\hline$\wedge$ & rq & $\varepsilon 1$ & 10 & عدم وجود المعرفة عن إضافة الكلمات لبناء & V \\
\hline$\varepsilon$ & rt & rt & ro & عدم معرفة معنى الكلمات حسب السياق & $\wedge$ \\
\hline
\end{tabular}

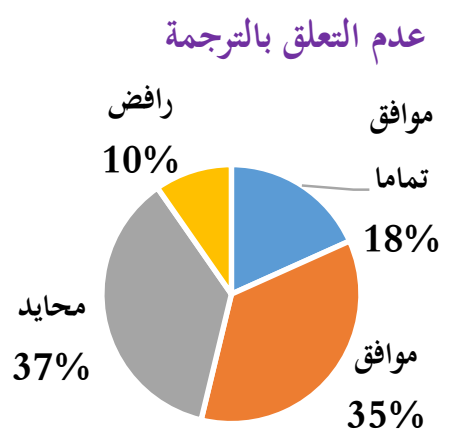

ا ل عدم التعلق بالترجمة : واجه الأغلبية من الطلبة الصعوبات عند ترجمة الجمل التاميلية إلى العربية في درجة متوسطة بسبب عدم التعلق والاشتغال في الترجمة وركن ثلاثة وثلاثين وافقوا في وجود هذه القضية، أما الأقل منهم سبعة عشر فوافقوا فيه تماما، في

الشكل ؛ : الشدم التعلق بالترجمة 
Muhammadu Sainulabdeen Zunoomy, Mohammed Cassim Sithy Shathifa

حين تسعة طلبة لم يقبلوا هذا السبب لمواجهة الصعوبات في الترجمة، ويبينه الشكل رقم (ع). r . عدم وجود المعرفة عن نظام الجمل في التاملية: لم يواجه الأغلبية من الطلبة الصعوبات

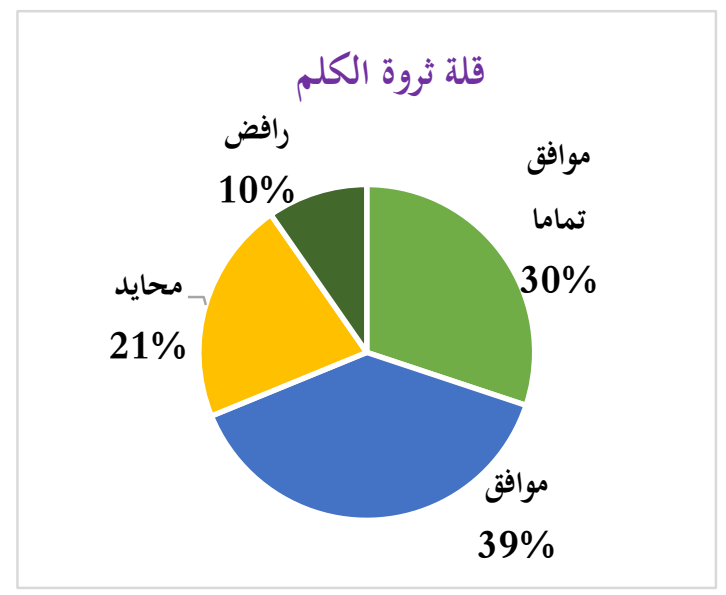

الشكل ه : عدم وجود المعرفة عن نظام الجمل في التاميلية

عدم وجود المعرفة عن نظام الجمل في الثاميلية

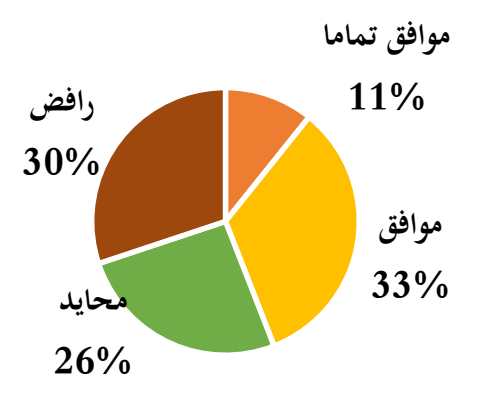

الشكل ج : قلة ثروة الكلمات عند ترجمة الجمل التاميلية إلى اللغة العربية في درجة متوسطة بسبب وجود المعرفة عن نظام الجمل في التاميلية ولكن واحدة وثلاثين وافقوا في وجود هذه القضية، أما الأقل منهم عشرة فوافقوا فيه تماما، في حين ثمانية وعشرون طالبا لم يقبلوا هذا السبب لمواجهة الصعوبات في الترجمة. وتؤيد هذه النتائج أن الطلبة واجهوا الصعب بدرجة أقل. ويبينه الشكل رقم (0). r. قلة ثروة الكلمات : واجه الأغلبية من الطلبة الصعوبات عند ترجمة الجمل التاميلية إلى اللغة العربية في درجة متوسطة بسبب قلة ثروة الكلمات ولكن ستة وثلاثين وافقوا في وجود هذه القضية، أما الأقل منهم ثمانبة وعشرون فوافقوا فيه تماما، في حين تسعة طلبة لم يقبلوا هذا السبب لمواجهة الصعوبات في الترجمة، ويبينه الشكل رقم (†).

ع. عدم وجود المعرفة عن قواعد اللغة العربية: واجه الأغلبية من الطلبة الصعوبات عند ترجمة الجمل التاميلية إلى اللغة العربية في درجة متوسطة بسبب عدم وجود المعرفة عن قواعد اللغة

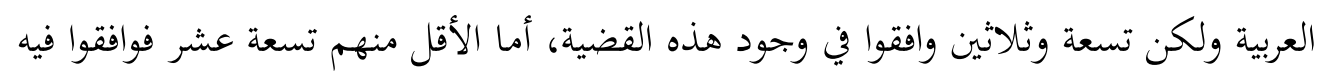


Muhammadu Sainulabdeen Zunoomy, Mohammed Cassim Sithy Shathifa

تماما، في حين ستة طلبة لم يقبلوا هذا السبب لمواجهة الصعوبات في الترجمة، ويبينه الشكل رقم

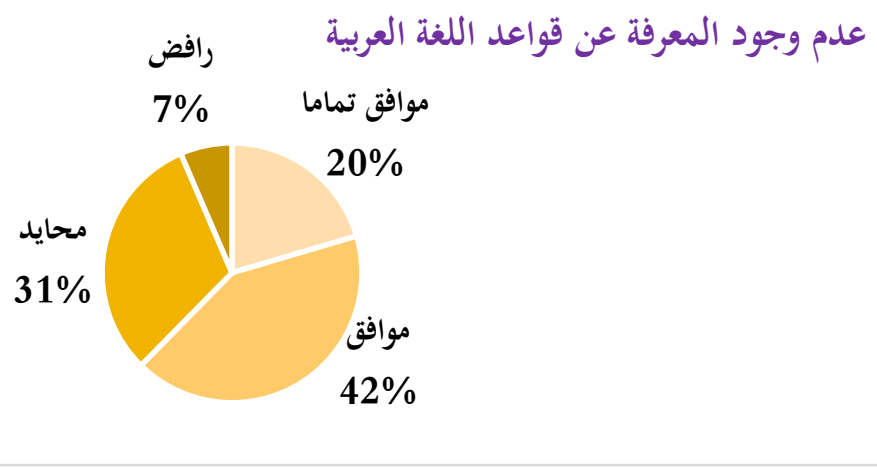

الشكل V: عدم وجود المعرفة عن قواعد اللغة العربية

الضعف في الكتابة بالعربية

ه. الضعف في الكتابة بالعربية: واجه

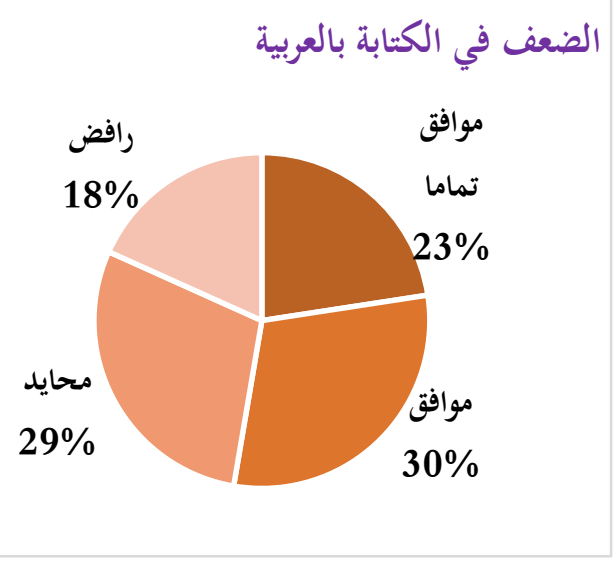

الأغلبية من الطلبة الصعوبات عند ترجمة الجمل التاميلية إلى اللغة العربية في درجة التهن متوسطة بسبب الضعف في الكتابة بالعربية

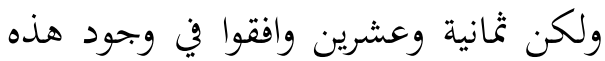

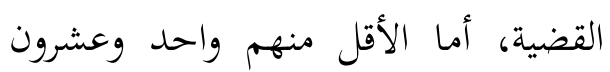
فوافقوا فيه تماما، في حين سبعة عشر طالبا

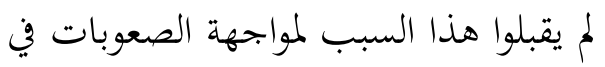
الترجمة، ويبينه الشكل رقم (^) 
Muhammadu Sainulabdeen Zunoomy, Mohammed Cassim Sithy Shathifa

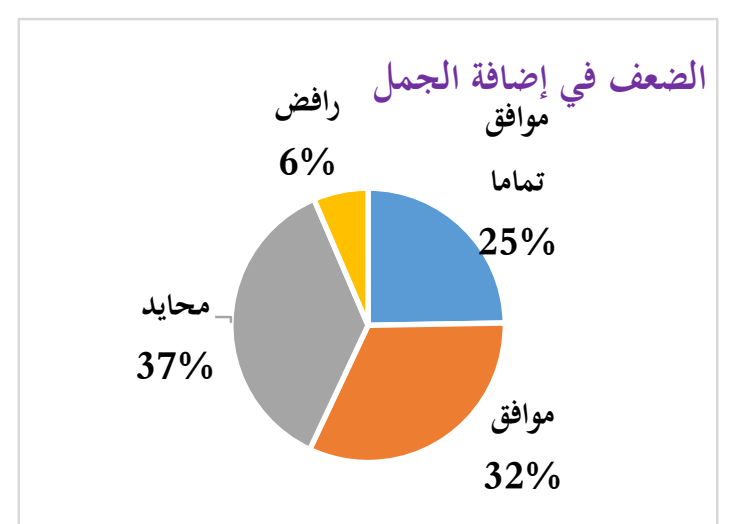

צ. الضعف في إضافة الجمل: واجه الأغلبية من الطلبة الصعوبات عند ترجمة الجمل التاميلية إلى اللغة العربية في درجة متوسطة بسبب الضعف في إضافة الجمل ولكن ثلاثين وافقوا في وجود هذه القضية، أما الأقل منهم ثلاثة وعشرون فوافقوا فيه الشكل 9: الضعف فى إضافة الجمل تماما، في حين ستة طلبة لم يقبلوا هذا السبب لمواجهة الصعوبات في الترجمة، ويبينه الشكل رقم (9).

V. عدم وجود المعرفة عن إضافة الكلمات لبناء الجمل الصحيحة: واجه الأغلبية من عدم وجود المعرفة عن إضافة الكلمات لبناء الجمل الصحيحة الطلبة الصعوبات عند ترجمة الجمل التاميلية إلى اللغة العربية في درجة متوسطة بسبب عدم وجود المعرفة عن إضافة

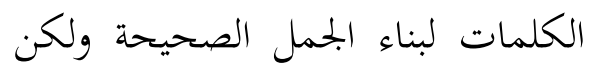
واحدا وأربعين وافقوا في وجود هذه

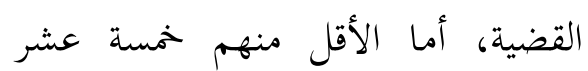
فوافقوا فيه تماما، في حين ثمانية طلبة لم الم يقبلوا هذا السبب لمواجهة الصعوبات في الشكل · ـ : عدم وجود المعرفة عن إضافة الكلمات لبناء الجمل الصحيحة الترجمة، ويبينه الشكل رقم (·) 
Muhammadu Sainulabdeen Zunoomy, Mohammed Cassim Sithy Shathifa

^. عدم معرفة معنى الكلمات حسب السياق: واجه الأغلبية من الطلبة الصعوبات عند ترجمة

عدم معرفة معنى الكلمات حسب السياق

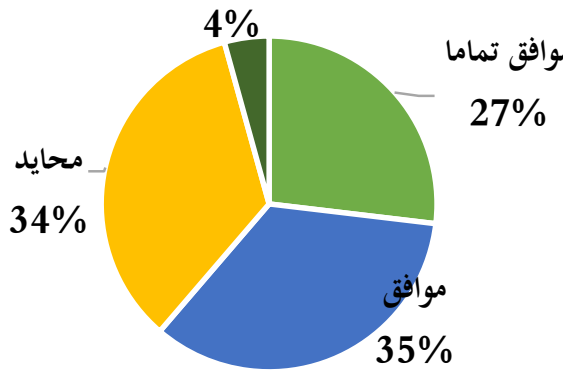

الشكل 11 1 : عدم معرفة معنى الكلمات حسب السياق
الجمل التاميلية إلى اللغة العربية في درجة

متوسطة بسبب عدم معرفة معنى الكلمات حسب السياق ولكن اثنين وثلاثين وافقوا في وجود هذه القضية، أما الأقل منهم خمسة وعشرون فوافقوا فيه تماما، في حين

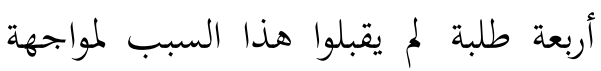
الصعوبات في الترجمة، ويبينه الشكل رقم

النتائج من تحليل الدراسات الميدانية:

يتناول هذا الموضوع النتائج لتحليل البيانات من الاستبانات والمقابلات الشخصية التي تمدف قياس الصعوبات والأخطاء التي يواجهها الطلبة عند ترجمة الجمل التاميلية إلى اللغة العربية. وعالج تحليل البيانات حول موضوعين رئيسين : الصعوبات والأخطاء في ترجمة الجمل التاميلية إلى اللغة العربية، والأسباب التي تؤدي إلى هذه الصعوبات والمشكلات في ترعين ريسين الجمات الحمل التاميلية إلى اللغة العربية.

الصعوبات والأخطاء في ترجمة الجمل التاملية إلى اللغة العربية:

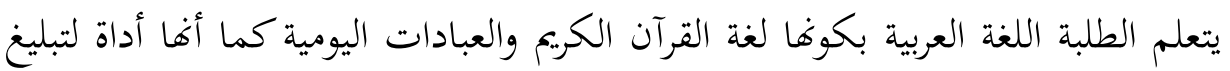
الدين الحنيف ووسيلة لفهم القرآن الكريم والسنة النبوية. وضّح تحليل الاستبانات أن الطلبة الذين يتعلمون اللغة العربية لغة أجنبية واجهوا الصعوبات عند ترجمة الجمل التاميلية إلى اللغة

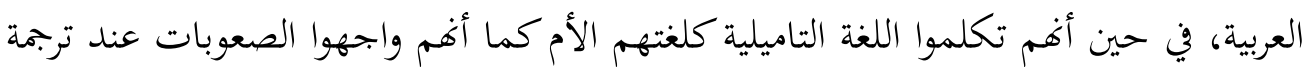


Muhammadu Sainulabdeen Zunoomy, Mohammed Cassim Sithy Shathifa

الجمل من التاميلية إلى العربية، لأن اللغة التاميلية لغتهم الأم مهما اللغة العربية لغتهم الأجنبية.

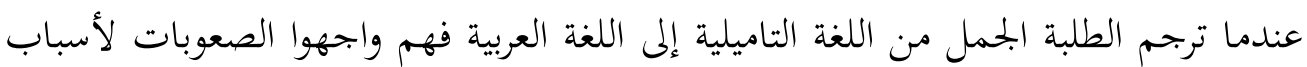
شتى وهي: عدم وجود المعرفة عن نظام الجمل في التاميلية، قلة ثروة الكلمات، عدم وجود المعرفة عن قواعد اللغة العربية، الضعف في الكتابة بالعربية، الضعف في إضافة الجمل، عدمد وجودود المعرفة عن إضافة الكلمات لبناء الجمل الصحيحة، عدم معرفة معنى الكلمات حسب السياق

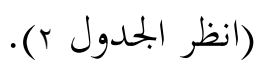

أما الأخطاء التي وقعت من الطلبة عند الترجمة من اللغة التاميلية إلى اللغة العربية فهي: الأخطاء النحوية، عدم استخدام الكلمات التاميلية المناسبة للكلمات العربية، عدم عناية نظام الجمل التاملية، عدم وجود المعرفة في إضافة الجمل التاملية. أن عدم استخدام الكلمات التاميلية المناسبة للكلمات العربية وإضافة الجمل بغير مناسبة وقع أكثر من غيرها. إن الطلبة ترجموا الجمل إصل البسيطة بوجه صحيح أكثر من الجمل المركبة والجمل المعقدة. لأغم لم يضيفوا الجمل العربية باستخدام أدوات العطف المناسبة (راجع الشكلين (، ب).

الأسباب التي أدت إلى الصعوبات والأخطاء في ترجمة الجمل التاميلية إلى اللغة العربية: وهي من قلة الرغبة في استخدام اللغة العربية والتاميلية صحيحا، قلة القراءة في اللغة التاميلية واللغة العربية، عدم الحرص في اطلاع اللغتين، التعلم حسب الحاجة، عدم الرغبات في تعلم اللغتين العربية والتاميلية، عدم وجود المعرفة عن قواعد اللغة العربية واللغة التاميلية، عدم التعلق بالترجمة، قلة التدريبات والتطبيقات في الترجمة، قلة ثروة الكلمات، عدم وجود المعرفة عن السياق اللغوي، الرغبة والاشتغال في الترجمة الحرفية (المصدر : الإجابات من المقابلات). خلاصة البحث:

إن تعليم اللغة العربية له أهمية قصوى للطلبة المسلمين من حيث أها لغة القرآن والعبادات اليومية. إن للطلبة الناطقين بالتاميلية علاقة وثيقة باللغة العربية من حيث أفم يتعلموفا من المدارس العربية والجامعات .وهكذا أهم يتعلموها لحصول شهادة البكاليوريوس. ولكن الطلبة لم 
Muhammadu Sainulabdeen Zunoomy, Mohammed Cassim Sithy Shathifa

يتقنوا في نظام الجمل العربية عند ترجمة الجمل التاميلية مهما تكلموا اللغة التاميلية كلغتهم الأم. إن الطلبة أحسنوا في ترجمة الجمل البسيطة من التاميلية إلى العربية، ولكنهم لم يعرفوا تماما عن

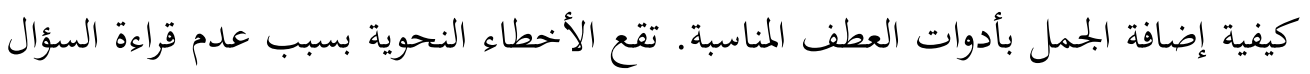
كاملا وفهمه كاملا. إن الطلبة عرفوا الكلمات في الجمل ولكنهم لم يعرفوا عن كيفية استخدامها حسب سياق الجمل.

الحلول المقترحة لهذه الصعوبات:

يجب على دارسي اللغة العربية لغة أجنبية الالتزام بالأمور التالية: تعلم اللغة العربية بوضوح مع الحرص الخالص، تعلم اللغة العربية بِبيانٍ من حيث أها لغة الهدف لناطقي اللغة التاملية، التدريب المستمر في بحال الترجمة، كثرة القراءة في اللغتين العربية والتاميلية، ازدياد المهارات اللغوية، العناية في نمو ثروة الكلمات، أن يهتم بالقواعد النحوية عند الترجمة، العناية بالأخطاء النحوية عند كتابة الجمل، أن يهتم بسياق الجمل، البعد عن الترجمة الحرفية.

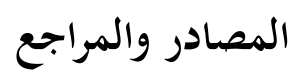

إبراهيم، خليل. (1...r). قواعد اللغة العربية للمتقدمين. عمان: الأهلية للنشر والتوزيع. ابن منظور، جمال الدين.(1990). لسان العرب. ط ط س. بيروت: دار الصادر.

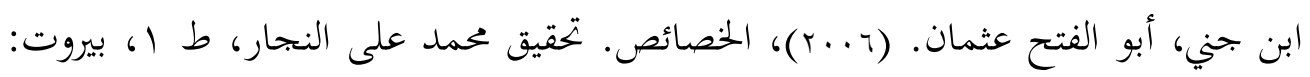
عالم الكتب.

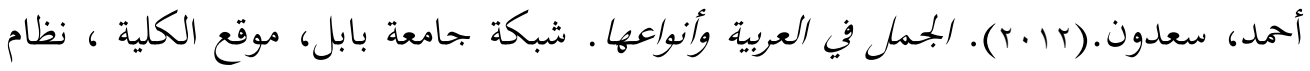
التعليم الالكتروني، مشاهدة المحاضرة. تم استرجاعه من www.uobabylon.edu.iq أحمد، علي الزين عبد النور ( (r • r). نظام الجملة في اللغتين العربية والفوروية (دراسة تقابلية).

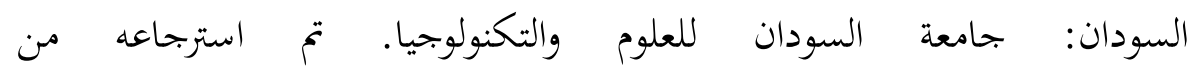
/repository.sustech.edu/handle/ 
Muhammadu Sainulabdeen Zunoomy, Mohammed Cassim Sithy Shathifa

أنيس، إبراهيم وعبد الحليم منتصر وعطية الصوالحي ومحمد خلف الله أحمد. (ع ..ب). المعتمم الوسيط. قطر :دار إحياء التراث الإسلامي.

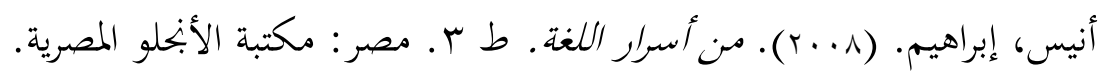

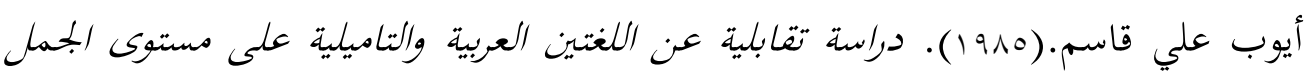
الصغرى. رسالة ماجستر غير منشورة في جامعة خرطوم.

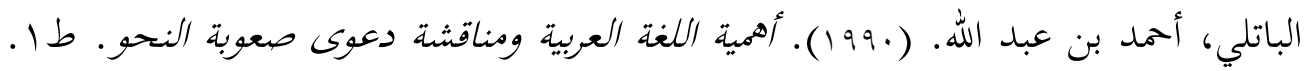
الرياض: دار الوطن للنشر.

$$
\text { بشر، كمال. (1979). دراسات في علم اللغة. مصر: دار المعارف. }
$$

الثقافة الإدارية. (v.....). مغهوم النظام لغة واصطلاحا. . من الموفع الإلكتروين ، منهل الثقافة التربوية. تمت المراجعة على الرابط https:// www. manhal.net/s/3696. الجرجاني، علي بن محمد.(1991). معجم التعريغات. القاهرة: دار الفضيلة للنشر والتوزيع والتصوير.

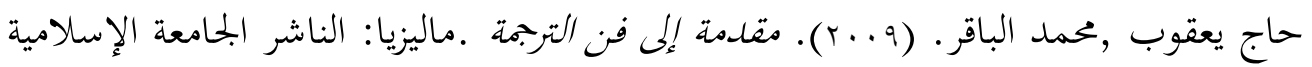

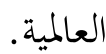

حسان، تمام. (1911). مناهج البحث في اللغة. القاهرة: دار الثقافة للنشر والتوزيع. حسن، عباس. (د.ت). النحو الوافي. ط هـ ا. القاهرة: دار المعارف.

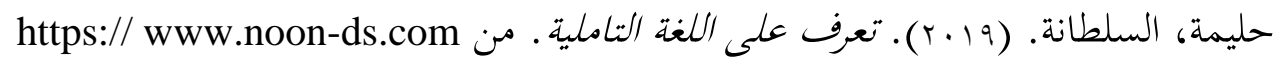
خرمة، نايف. (1911). اللغات الأجنبية، تعليمها وتعلمها . عالم المعرفة. رواي، صلاح محمد مصطفى. (199r). فقه اللغة ونصائص العببية وطرائق نموها. ط لـ ا. القاهرة: مكتبة الزهراء.

السحيباني، سليمان بن عمر .(10 (1).أسماء الإثارة في العربية و الإنجليزية: دراسة تقابلية. بملة

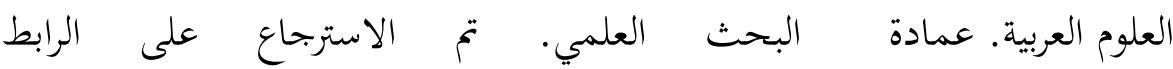
.www.imamu.edu.sa سعدية، نعيمة. (11.r). الجملة في الدراسات اللغوية. . بسكرة: جامعة محمد خيضر. 
Muhammadu Sainulabdeen Zunoomy, Mohammed Cassim Sithy Shathifa

السمرة، محمود، والموسى غاد.(1910). نظام الجمل والإعراب. سلطنة عمان: وازرة التربية والتعليم وشؤون الشباب.

صادفة بنت محمد قاسم ، شحيقة فرون بنت عبد الرحيم.( (10 ب). أدوات الربط واستخدامها

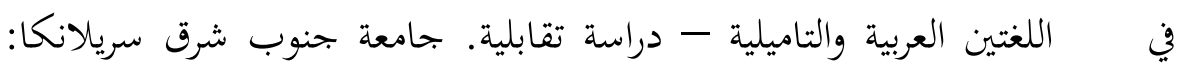
مؤتمر كلية الآداب والثقافة.

صالح، ناصر.(r(r). الجملة العربية والجملة الإنحليزية - دراسة تقابلية.السعودية:بجلة التربية

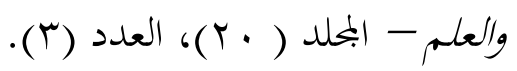

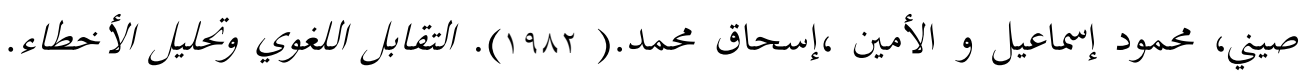
السعودية: الناشر عمادة شؤون المكتبات - جامعة الملك سعود.

عبادة، محمد إبراهيم. (911 (1). الجملة العربية دراسة نحوية. الاسكندرية: منشأة المعارف. عبد عبد الله. (YlV). r. دراسة تقابلية بين اللغة العربية و لغة الهوسا على مستوى الضمائر. رسالة ماجستير ، الآداب في اللغة العربية، تخصص علم اللغة. جامعة الجزيرة: كلية

$$
\text { التربية حنتوب - قسم اللغة العربية والدراسات الإسلامية. }
$$

عبد اللطيف، محمد حماسة. (r...r). بناء الجملة العربية. ط ا ـ القاهرة: دار غريب. عبد النور، جبور . (ع 9 1 ). المعجم الأدبي. بيروت: دار العلم للملايين. العصيلي، عبد العزيز بن إبراهيم.(1) (†). أساسيات تعليم اللغة العربية للناطقين بلغات أخرى.

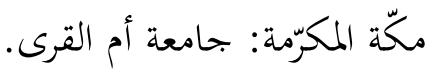

علي الجحارم ومصطفى أمين.(1999) . النحو الواضح في قواعد اللغة العربية . القاهرة: دار المعارف.

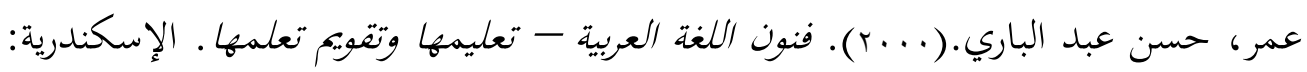
مركز الإسكندرية للكتاب.

قدّور ، أحمد محمد.(1999) . مبادئ اللسانيات. ط ب ب. دمشق: دار الفكر.

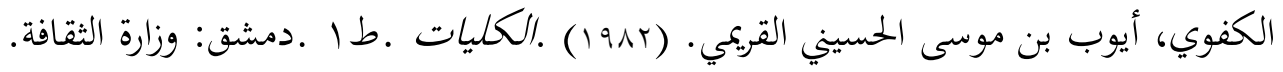
المبارك، مازن.(1911). فقه اللغة وخصائص العربية. بيروت: دار الفكر. 
Muhammadu Sainulabdeen Zunoomy, Mohammed Cassim Sithy Shathifa

متولي، عبد الله عبد الحافظ. (1990). الترجمة - أصولها ومبادئها وتطبيقاتها .مصر: دار النشر للجامعات المصرية - مكتبة الوفاء.

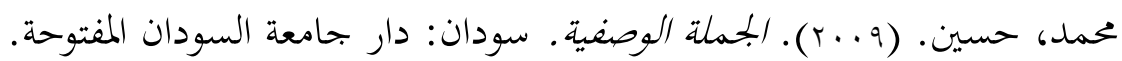
محمد، عادل خلف عبد الجواد.(\$ 199). اللغة والبحث اللغوي. بيروت : مكتبة الآداب. تم https://www .startimes. com/l=8413922.t=اساسترجاع على الرابط

المخزومي، مهدي محمد صالح. (1914). في النحو العربي نقل وتوجيه. ط ط ب. بيروت: دار

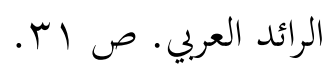

المسدي، عبد السلام.(1991). من الكلمة إلى الجملة بحث في منهج النحاة. مؤسسات عبد الكريم عبد الله.

مصلح، حسام. (7 ( +r).نظام الجملة في اللغتين: العربية، والانجليزية. دنيا الوطن. تم الاسترجاع https://pulpit.alwatanvoice.com. على الرابط معروف، فريد. (·.+r). الجملة الفعلية في اللغة العربية و Kalimat Verbal في الغنة الإنلونيسية . جاكرتا: جامعة شريف هداية الله الإسلامية الحكومية.

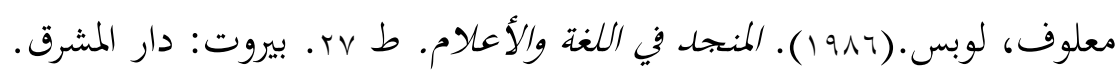

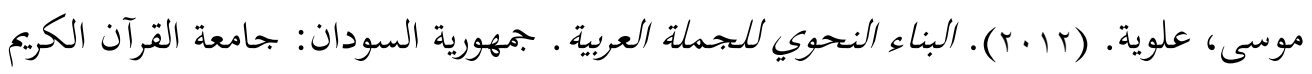
والعلوم الإسلامية.

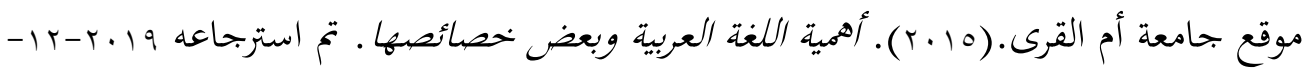

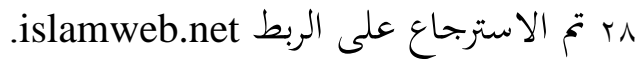

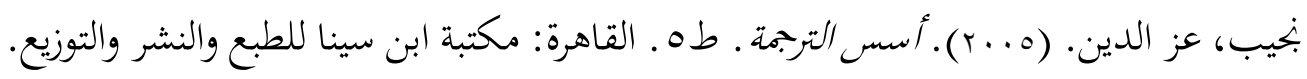

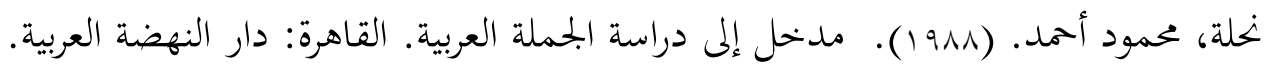

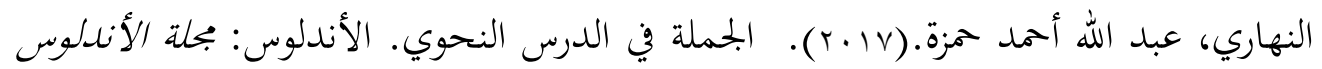
للعلوم الإنسانية والاجتماعية. 
Muhammadu Sainulabdeen Zunoomy, Mohammed Cassim Sithy Shathifa

$$
\begin{aligned}
& \text { النواصرة، ناصر محمود صالح · (rا.r). الجملة العببية و الجملة الإبجليزية - درامة تقابلية. } \\
& \text { السعودية: بحلة التربية والعلم. } \\
& \text { هاشم، عبد الوهاب.(919 19). محاضرات في تلدريس اللغة العربية . أسيوط: مطبعة سمكة. }
\end{aligned}
$$

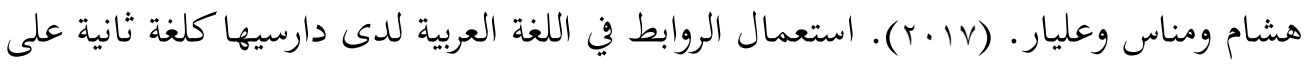

$$
\begin{aligned}
& \text { مستوى الجامعة نموذجا طلاب السنة الأولى من جامعة جنوب شرق سريلانكا.جامعة } \\
& \text { جنوب شرق سريلانكا: Arts Research Session. }
\end{aligned}
$$

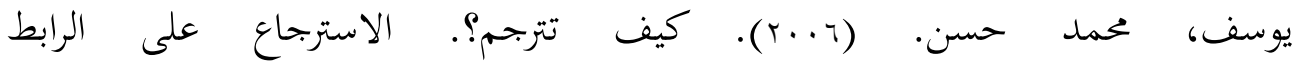

$$
\begin{aligned}
& \text { http://saaid.net/Doat/hasn/index.htm }
\end{aligned}
$$

Aliyar, ABM., Shathifa, MCS. \& Farween, ARFS. (2014). The role of context in determining the meaning of a word from Arabic to Tamil translation. South Eastern University of Sri Lanka: 5th International Symposium.

Breedlove, Cliff. (n.d) .Arabic and English Sentence Patterns - A Comparative Guide. Portland: State University Library.

Karunakaran, k. (2000). Simplified Grammar of Tamil. Chennai: Suvita Publishers.

Kharma, Nayef.(1983). A Contrastive Analysis of the Use of Verb Forms in English and Arabic. Heidelberg: J. Groos.

Mohammed EMM, Habeebullah MT. (2015). Teaching of Arabic Grammar: Problems and Solutions. South Eastern University of Sri Lanka: $5^{\text {th }}$ International Symposium.

Muttuch Chanmuham. (1998). Ikkala Mozhieyal. Chennai: Mullai Nilayam.

Nuhman, M. A. (2006). Adippadai Tamil Ilakkanam. Colombo: Poobalasinga Puththahasaalai. 
Muhammadu Sainulabdeen Zunoomy, Mohammed Cassim Sithy Shathifa

Pansaadsaram, S. V. (2002). Tamil Ilakkanap Poonga. Inuval North: Ponneluththup Pathippaham.

Siththarthan, Singapore. (2003). Ilahu Tamilil Inikkum Tamil Ilakkanam.

Chennai: Narmatha Publisher

Sivanana Sundaram, I.K. (2004). Tamil Ilakkiya Ilakkanap Pezhai. Colombo: Thenral Publication.

Zvelebil, Kamil (1973). The Smile of Murugan, BRILL. 